\section{Chikungunya in Brazil: Rheumatologists on the Front Line}

\section{To the Editor:}

Chikungunya virus (CHIKV) is an enveloped, spherical single-stranded RNA virus about $60-70 \mathrm{~nm}$ in diameter, belonging to the alphavirus genus and family Togaviridae. CHIKV is transmitted by Aedes species mosquito vectors and causes an acute disabling illness characterized by high fever and severe arthralgia called chikungunya fever $(\mathrm{CHIKF})^{1,2}$. The word chikungunya means "the one which bends up" in the Makonde dialect of Tanzania; it refers to severe joint pain characteristic of acute disease ${ }^{2}$. CHIKV was isolated in Tanzania in 1952 during a dengue-like outbreak ${ }^{1,2,3}$. Since then, CHIKV has caused outbreaks in Africa, Asia, the Indian Ocean region, and Europe, with severe medical and economic effects ${ }^{1}$. In December 2013, CHIKV was reported in the Americas on the island of Saint Martin. Since then, more than 2.9 million cases have been reported in 45 countries in North, Central, and South America ${ }^{3}$.

The history of CHIKF in the Americas is probably at least 200 years $\mathrm{old}^{4}$. For centuries, CHIKV existed in forested regions of eastern Africa in an enzootic life cycle that affected arboreal mosquito vectors and nonhuman primates $^{5}$. Phylogenetic analysis suggests that there has been periodic emergence of an epidemic life cycle; this includes the spreading beyond Africa to the new world in ships containing infected individuals, Aedes mosquitoes, and stagnant water ${ }^{6}$.

Brazil is a tropical and subtropical country of 210 million people. It is infested in every state with Aedes mosquitoes. Major outbreaks in Brazil of dengue and Zika virus infection have been widely reported. They result from the tropical environment, urban crowding, and challenges in vector control. The first locally transmitted CHIKV infection in Brazil occurred in September 2014 in the state of Amapá (North), followed by an outbreak in the state of Bahia (Northeast). In the same year, 1425 autochthonous cases of CHIKF were confirmed by clinical-epidemiological and/or laboratory criteria. By the end of 2015, 38,332 suspected autochthonous CHIKF cases were reported and of these, 13,236 were confirmed, with 6 deaths. In 2016, there were 263,598 suspected CHIKF cases in 2700 cities and 145,059 confirmed cases, which resulted in more than 200 deaths. As of September 2017, 171,930 suspected cases and 121,734 confirmed cases were reported $^{7,8}($ Table 1$)$.

As of September 2017, among the 5 Brazilian federal regions, the Northeast had the highest number of reported cases and confirmed deaths, with $>400,000$ cases and 278 deaths ${ }^{9}$. Ceará, in the Northeast, reported $>149,760$ probable CHIKF cases. In addition, the epidemic is spreading toward the Southeast and North regions (Figure 1).

Of the 3 CHIKV genotypes, the Asian one, reported in the Caribbean, was detected in the state of Amapá, and the East/Central/South African (ECSA) genotype, which had not previously been detected in the Americas, was confirmed in the state of Bahia. Brazil therefore harbors the highest diversity of CHIKV in the Americas ${ }^{10}$.

In Brazil and throughout the Americas, CHIKF significantly affects rheumatology practice. Following acute CHIKF, up to $60 \%$ of patients develop arthralgia, arthritis, myalgia, and fatigue, lasting up to 3 years ${ }^{1}$.
Physicians in endemic areas are confronted with large numbers of patients with chronic disabling arthritis. The most severely affected individuals are likely to be referred to rheumatologists. There is a lack of consensus about how these patients should be treated, and many drugs, including nonsteroidal antiinflammatory drugs, glucocorticoids, disease-modifying antirheumatic drugs (methotrexate, hydroxychloroquine, sulfasalazine), and biological drugs have been used to treat CHIKF chronic arthritis with limited success or evidenced-based support ${ }^{1,2}$. There is scientific interest in CHIKV vaccine development, but commercialization seems unlikely in the near future.

CHIKF is an emerging viral infection. Chronic chikungunya arthritis is an emerging rheumatic disease. The vectors of CHIKF (Aedes albopictus and Aedes aegypti) are found on every continent except Antarctica ${ }^{2}$. The Brazilian chikungunya epidemic, like previous epidemics in Africa, India, and elsewhere, has created widespread chronic rheumatic disability that is likely to recur in new epidemics in the near future. Brazil has just over 2000 rheumatologists, a limited number to manage nearly half a million reported chikungunya arthritis cases in the past 3 years ${ }^{9}$. In Brazil, studies on the effect of the disease, its characteristics, and treatment are needed. Rheumatologists in Brazil are on the front line now and need new resources to combat this infectious arthritis. It is incumbent on the entire rheumatology community to take up this challenge.

No ethics approval is required according to the authors' institutions (Faculty of Medicine Estácio of Juazeiro do Norte, and Yale University School of Medicine). No individual human subjects were studied or exposed to any risk.

J. KENNEDY AMARAL, MD, Faculty of Medicine Estácio of Juazeiro do Norte, Juazeiro do Norte, Brazil; ROBERT T. SCHOEN, MD, MBA, Clinical Professor of Medicine, Section of Rheumatology, Yale University School of Medicine, New Haven, Connecticut, USA. Address correspondence to Dr. R. Schoen, 60 Temple St., Suite 6A, New Haven, Connecticut 06510, USA. E-mail: robert.schoen@yale.ed

\section{REFERENCES}

1. Thiberville SD, Moyen N, Dupuis-Maguiraga L, Nougairede A, Gould EA, Roques P, et al. Chikungunya fever: epidemiology, clinical syndrome, pathogenesis and therapy. Antiviral Res 2013;99:345-70.

2. Presti AL, Lai A, Cella E, Zehender G, Ciccozzi M. Chikungunya virus, epidemiology, clinics and phylogenesis: A review. Asian Pac J Trop Med 2014;7:925-32.

3. Yactayo S, Staples JE, Millot V, Cibrelus L, Ramon-Pardo P. Epidemiology of chikungunya in the Americas. J Infect Dis 2016;214: S441-5.

4. Halstead SB. Reappearance of chikungunya, formerly called dengue, in the Americas. Emerg Infect Dis 2015; 21:557-61.

5. Weaver SC, Lecuit M. Chikungunya virus and the global spread of a mosquito-borne disease. N Engl J Med 2015;372:1231-9.

6. Volk SM, Chen R, Tsetsarkin KA, Adams AP, Garcia TI, Sall AA, et al. Genome-scale phylogenetic analyses of chikungunya virus

Table 1. Number of probable CHIKF cases, confirmed deaths, and incidence per 100,000 population ${ }^{8,9}$.

\begin{tabular}{|c|c|c|c|c|c|c|c|c|c|c|c|c|}
\hline \multirow[t]{2}{*}{ Region } & \multicolumn{4}{|c|}{ No. Cases } & \multicolumn{4}{|c|}{ No. Deaths } & \multicolumn{4}{|c|}{ Incidence (per 100,000 population) } \\
\hline & 2014 & 2015 & 2016 & $2017 *$ & 2014 & 2015 & 2016 & $2017 *$ & 2014 & 2015 & 2016 & $2017^{*}$ \\
\hline North & 1516 & 1463 & 7560 & 14,888 & 0 & 0 & 0 & 5 & 8.9 & 8.4 & 43.3 & 84.1 \\
\hline Northeast & 16,013 & 34,169 & 229,157 & 130,910 & 0 & 5 & 188 & 85 & 28.5 & 60.4 & 405.2 & 230.0 \\
\hline Southeast & 9 & 420 & 23,539 & 22,789 & 0 & 1 & 14 & 8 & 0.01 & 0.5 & 27.5 & 26.4 \\
\hline South & 13 & 78 & 1689 & 262 & 0 & 0 & 0 & 0 & 0.04 & 0.3 & 5.8 & 0.9 \\
\hline Central & 214 & 124 & 1653 & 3081 & 0 & 0 & 2 & 1 & 1.4 & 0.8 & 10.7 & 19.7 \\
\hline Total & 17,765 & 36,254 & 263,598 & 171,930 & 0 & 6 & 204 & 99 & 8.7 & 17.7 & 128.9 & 83.4 \\
\hline
\end{tabular}

*Up to September 2017. CHIKF: chikungunya fever. 


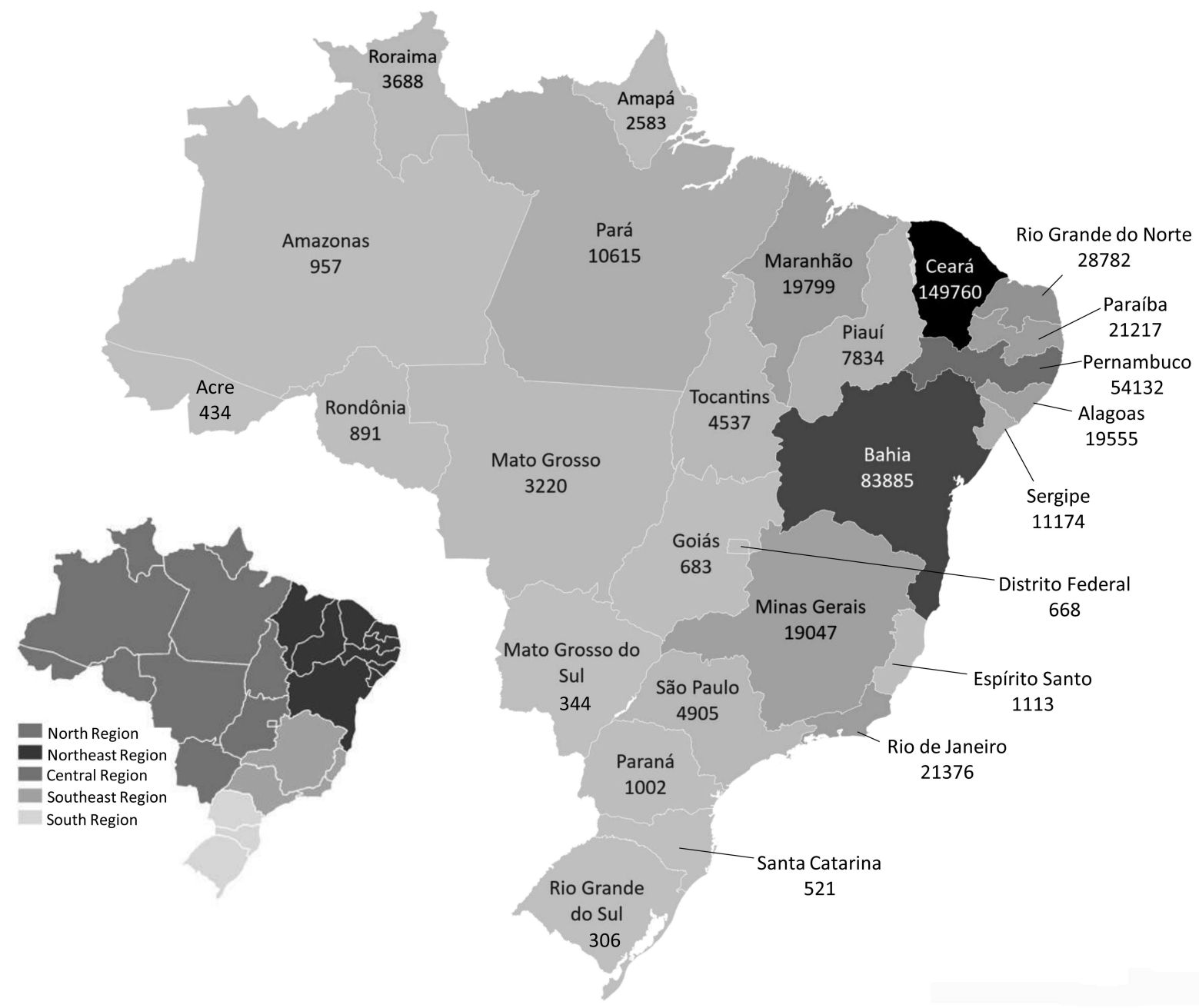

Série1

Figure 1. Map of Brazil illustrating the number of CHIKV cases distributed among the 5 Brazilian federal regions. The Northeast had the highest number of reported cases and confirmed deaths; the epidemic is spreading to the North and the Southeast. States with the highest number of cases in the North, Northeast, Central, Southeast, and South regions were respectively Pará (10,615), Ceará (149,750), Tocantins (4537), Rio de Janeiro $(21,376)$, and Paraná (1002). Série1 refers to the range of CHIKV cases reported in Brazil. Data from the Ministry of Health of Brazil, Department of Health Surveillance, Boletim Epidemiológico (cases of dengue and chikungunya fever until Epidemiological Week 35, 2017) ${ }^{9}$. CHIKV: chikungunya virus.

reveal independent emergences of recent epidemics and various evolutionary rates. J Virol 2010;84:6497-504.

7. Azevedo Rdo S, Oliveira CS, Vasconcelos PF. [Chikungunya risk for Brazil]. [Article in Portuguese] Rev Saúde Pública 2015;49:58.

8. Ministry of Health of Brazil. Department of Health Surveillance. Boletim Epidemiológico. [Monitoring of cases of dengue and chikungunya fever until Epidemiological Week 48, 2015]. [Internet. Accessed March 21, 2018. In Portuguese.] Available from: portalarquivos.saude.gov.br/images/pdf/2016/janeiro/07/ 2015-svs-be-pncd-se48.pdf

9. Ministry of Health of Brazil. Department of Health Surveillance. Boletim Epidemiológico. Monitoring of cases of dengue and chikungunya fever until Epidemiological Week 35, 2017. [Internet. Accessed March 21, 2018.] Available from: portalarquivos.saude.gov.br/images/pdf/2017/setembro/15/ 2017-028-Monitoramento-dos-casos-de-dengue-

febre-de-chikungunya-e-febre-pelo-virus-Zika-ate-a-SemanaEpidemiologica-35.pdf

10. Charlys da Costa A, Thézé J, Komninakis SCV, Sanz-Duro RL, Felinto MRL, Moura LCC, et al. Spread of chikungunya virus East/Central/South African genotype in Northeast Brazil. Emerg Infect Dis 2017;23:1742-4.

J Rheumatol 2018;45:10; doi:10.3899/jrheum.171237 